\title{
Scattering Analysis of Electromagnetic Materials Using Fast Dipole Method Based on Volume Integral Equation
}

\author{
Xiaoqiao Deng, Changqing Gu, Bingzheng Xu, Zhuo Li, and Xinlei Chen \\ College of Electronic and Information Engineering, Nanjing University of Aeronautics and Astronautics, \\ Nanjing 210016, China \\ Correspondence should be addressed to Xiaoqiao Deng; xiaoqiaodeng@gmail.com
}

Received 28 November 2012; Accepted 16 January 2013

Academic Editor: Ahmed A. Kishk

Copyright (C) 2013 Xiaoqiao Deng et al. This is an open access article distributed under the Creative Commons Attribution License, which permits unrestricted use, distribution, and reproduction in any medium, provided the original work is properly cited.

The fast dipole method (FDM) is extended to analyze the scattering of dielectric and magnetic materials by solving the volume integral equation (VIE). The FDM is based on the equivalent dipole method (EDM) and can achieve the separation of the field dipole and source dipole, which reduces the complexity of interactions between two far groups (such as group $i$ and group $j$ ) from $O\left(N_{i} N_{j}\right)$ to $O\left(N_{i}+N_{j}\right)$, where $N_{i}$ and $N_{j}$ are the numbers of dipoles in group $i$ and group $j$, respectively. Targets including left-handed materials (LHMs), which are a kind of dielectric and magnetic materials, are calculated to demonstrate the merits of the FDM. Furthermore, in this study we find that the convergence may become much slower when the targets include LHMs compared with conventional electromagnetic materials. Numerical results about convergence characteristics are presented to show this property.

\section{Introduction}

The electromagnetic materials have gained wide attention and interest for a long time. The method of moments (MoM) based on the volume integral equation (VIE) [1] is one of the most popular numerical methods to analyze the scattering of the electromagnetic materials. However, the conventional MoM needs $O\left(N^{2}\right)$ CPU time and memory requirement for an iterative solver. The complexity is very expensive for a personal computer with limited resources. Fortunately, many methods have been proposed in the past decades to deal with this problem, such as multilevel fast multipole algorithm (MLFMA) [2-4], the adaptive integral method (AIM) [5, 6], and the conjugate gradient-fast Fourier transform (CG-FFT) [7]. These methods improve the efficiency (computer storage and solution time) of the conventional MoM.

Recently, the equivalent dipole method (EDM) [8-10] is proposed to simplify the impedance matrix element filling procedure for the MoM, in which the Schaubert-WiltonGlisson (SWG) [11] elements are equivalent to an infinitely small dipole. However, it cannot save memory and reduce iterative time. More recently, the fast dipole method (FDM) $[12,13]$ is developed to mitigate the problem of the EDM, which can efficiently calculate the interactions between far groups. It reduces the complexity of interactions between two far groups, such as group $i$ and group $j$, from $O\left(N_{i} N_{j}\right)$ to $O\left(N_{i}+N_{j}\right)$, where $N_{i}$ and $N_{j}$ are the numbers of the dipoles in groups $i$ and $j$, respectively.

In this work, we extended the FDM to analyze the scattering of dielectric and magnetic materials by solving the volume integral equation (VIE). These materials not only contain the conventional materials but also contain the left-handed materials (LHMs) and LHM-RHM (righthanded material) composite materials. We find that it usually converges slowly when solving the targets involving LHMs.

\section{Volumetric Integral Equations for Electromagnetic Materials}

Consider an arbitrarily shaped 3D scattering electromagnetic object illuminated by a plane wave incident fields $\left(\mathbf{E}^{i}, \mathbf{H}^{i}\right)$, the permittivity and permeability of the object are characterized by $\varepsilon(\mathbf{r})$ and $\mu(\mathbf{r})$. The background space surrounding the dielectric targets with region $V$ is free space with $\varepsilon_{0}=$ $\mu_{0}=1$. According to the equivalence principle and boundary 
conditions, the total electromagnetic field $(\mathbf{E}, \mathbf{H})$ can be described as follows:

$$
\begin{gathered}
\mathbf{E}=\mathbf{E}^{i}+\mathbf{E}^{s}\left(\mathbf{J}_{v}^{e}\right)+\mathbf{E}^{s}\left(\mathbf{J}_{v}^{m}\right), \\
\mathbf{H}=\mathbf{H}^{i}+\mathbf{H}^{s}\left(\mathbf{J}_{v}^{m}\right)+\mathbf{H}^{s}\left(\mathbf{J}_{v}^{e}\right),
\end{gathered}
$$

where $\mathbf{E}^{s}$ and $\mathbf{H}^{s}$ denote the scattering electromagnetic fields. $\mathbf{J}_{v}^{e}(\mathbf{r})$ and $\mathbf{J}_{v}^{m}(\mathbf{r})$ denote equivalent volumetric electric and magnetic currents, respectively. $\mathbf{E}^{s}\left(\mathbf{J}_{v}^{e}\right), \mathbf{E}^{s}\left(\mathbf{J}_{v}^{m}\right), \mathbf{H}^{s}\left(\mathbf{J}_{v}^{m}\right)$, and $\mathbf{H}^{s}\left(\mathbf{J}_{v}^{e}\right)$ are the scattered electric field and magnetic field produced by the equivalent volume currents $\mathbf{J}_{v}^{e}$ and $\mathbf{J}_{v}^{m}$ and can be found in [10]. $\mathbf{J}_{v}^{e}(\mathbf{r})$ and $\mathbf{J}_{v}^{m}(\mathbf{r})$ can be expressed as

$$
\begin{aligned}
& \mathbf{J}_{v}^{e}(\mathbf{r})=j \omega \kappa^{e}(\mathbf{r}) \cdot \mathbf{D}(\mathbf{r}), \\
& \mathbf{J}_{v}^{m}(\mathbf{r})=j \omega \kappa^{m}(\mathbf{r}) \cdot \mathbf{B}(\mathbf{r}),
\end{aligned}
$$

where $\kappa^{e}(\mathbf{r})$ and $\kappa^{m}(\mathbf{r})$ are the contrast ratios, and

$$
\begin{aligned}
\kappa^{e}(\mathbf{r}) & =1-\varepsilon_{r}^{-1}(\mathbf{r}), \\
\kappa^{m}(\mathbf{r}) & =1-\mu_{r}^{-1}(\mathbf{r}) .
\end{aligned}
$$

The region $V$ is discretized into small tetrahedral elements. Then, the unknown volume currents $\mathbf{J}_{v}^{e}$ and $\mathbf{J}_{v}^{m}$ can be expanded by a set of SWG basis functions [11] as

$$
\begin{gathered}
\mathbf{J}_{v}^{e}(\mathbf{r})=\sum_{n=1}^{N_{v}} I_{n}^{e} \kappa_{n}^{e} \mathbf{f}_{n}(\mathbf{r}), \\
\mathbf{J}_{v}^{m}(\mathbf{r})=\eta \sum_{n=1}^{N_{v}} I_{n}^{m} \kappa_{n}^{m} \mathbf{f}_{n}(\mathbf{r}),
\end{gathered}
$$

where $I_{n}^{e}$ and $I_{n}^{m}$ are the unknown expansion coefficients for the electric and magnetic currents, respectively. $\kappa_{n}^{e}$ and $\kappa_{n}^{m}$ are the contrast ratios in the $n$th SWG basis function. $\mathbf{f}_{n}$ denotes the $n$th volume basis function defined in two adjoining tetrahedrons in the volume meshes associated with common face $n . N_{v}$ is the number of faces of the tetrahedral meshes. $\eta=\sqrt{\mu_{0} / \varepsilon_{0}}$ is the impedance of the free space. Using the Galerkin's method and testing the resultant integral equations with a set of testing functions, as a result, the integral equations are converted into a matrix equation, and can be written in the matrix form as

$$
\left[\begin{array}{ll}
\mathbf{Z}^{E E} & \mathbf{Z}^{E M} \\
\mathbf{Z}^{M E} & \mathbf{Z}^{M M}
\end{array}\right]\left[\begin{array}{c}
\mathbf{I}^{E} \\
\mathbf{I}^{M}
\end{array}\right]=\left[\begin{array}{c}
\mathbf{V}^{E} \\
\mathbf{V}^{M}
\end{array}\right] .
$$

The impedance elements of the submatrices $\mathbf{Z}^{E E}, \mathbf{Z}^{E M}, \mathbf{Z}^{M E}$, and $\mathbf{Z}^{M M}$ are represented as

$$
\begin{gathered}
Z_{m n}^{e e}=\left\langle\mathbf{f}_{m}, \mathbf{E}-\mathbf{E}^{s}\left(\mathbf{J}_{v n}^{e}\right)\right\rangle, \\
Z_{m n}^{e m}=\left\langle\mathbf{f}_{m},-\mathbf{E}^{s}\left(\mathbf{J}_{v n}^{m}\right)\right\rangle, \\
Z_{m n}^{m e}=\left\langle\eta \mathbf{f}_{m},-\mathbf{H}^{s}\left(\mathbf{J}_{v n}^{e}\right)\right\rangle, \\
Z_{m n}^{m m}=\left\langle\eta \mathbf{f}_{m}, \mathbf{H}-\mathbf{H}^{s}\left(\mathbf{J}_{v n}^{m}\right)\right\rangle,
\end{gathered}
$$

where $\mathbf{J}_{v n}^{e}$ and $\mathbf{J}_{v n}^{m}$ denote equivalent volume electric and magnetic currents corresponding to the $n$th SWG element. The elements in voltage vectors $\mathbf{V}^{E}$ and $\mathbf{V}^{M}$ are

$$
\begin{gathered}
V_{m}^{e}=\left\langle\mathbf{f}_{m}, \mathbf{E}^{i}\right\rangle, \\
V_{m}^{m}=\left\langle\eta \mathbf{f}_{m}, \mathbf{H}^{i}\right\rangle .
\end{gathered}
$$

\section{Fast Dipole Method for Electromagnetic Materials}

The FDM is an efficient way to solve the VIE, which is based on the EDM. In the EDM, each SWG element can be approximated as an infinitely small dipole with an equivalent moment. Referring to [10], the $n$th volume electric dipole moment $\mathbf{m}_{n}^{e}$ and magnetic dipole moment $\mathbf{m}_{n}^{m}$ can be represented as

$$
\begin{gathered}
\mathbf{m}_{n}^{e}=a_{n} \kappa_{n}^{e-}\left(\mathbf{r}_{n}^{c-}-\mathbf{r}_{n s}^{c}\right)+a_{n} \kappa_{n}^{e+}\left(\mathbf{r}_{n s}^{c}-\mathbf{r}_{n}^{c+}\right), \\
\mathbf{m}_{n}^{m}=a_{n} \kappa_{n}^{m-}\left(\mathbf{r}_{n}^{c-}-\mathbf{r}_{n s}^{c}\right)+a_{n} \kappa_{n}^{m+}\left(\mathbf{r}_{n s}^{c}-\mathbf{r}_{n}^{c+}\right),
\end{gathered}
$$

where $\mathbf{r}_{n}^{c \pm}$ is the position vector of the centroid of $T_{n}^{ \pm}$and $\mathbf{r}_{n s}^{c}$ is the position vector of the centroid of the common face of the $T_{n}^{ \pm} \cdot a_{n}$ is the area of the $n$th common face associated with $T_{n}^{ \pm} \cdot \kappa_{n}^{e \pm}$ and $\kappa_{n}^{m \pm}$ stand for the contrast ratio of $T_{n}^{ \pm}$. The impedance matrix elements in (6) can be calculated by the EDM:

$$
\begin{gathered}
Z_{m n}^{u u}=j k \eta \mathbf{m}_{m}^{\prime} \cdot \overline{\mathbf{G}}(\mathbf{R}) \cdot \mathbf{m}_{n}^{u}, \quad u=e, m, \\
Z_{m n}^{m e}=j k \eta \mathbf{m}_{m}^{\prime} \cdot \mathbf{G}(\mathbf{R}) \times \mathbf{m}_{n}^{e}, \\
Z_{m n}^{e m}=-j k \eta \mathbf{m}_{m}^{\prime} \cdot \mathbf{G}(\mathbf{R}) \times \mathbf{m}_{n}^{m},
\end{gathered}
$$

where

$$
\begin{aligned}
\overline{\mathbf{G}}(\mathbf{R})=\frac{e^{-j k R}}{4 \pi R}\left[\overline{\mathbf{I}}\left(1+\frac{1}{j k R}+\frac{1}{(j k R)^{2}}\right)\right. \\
\left.-\widehat{\mathbf{R} \widehat{R}}\left(1+\frac{3}{j k R}+\frac{3}{(j k R)^{2}}\right)\right], \\
\mathbf{G}(\mathbf{R})=\frac{e^{-j k R}}{4 \pi R}\left(1+\frac{1}{j k R}\right) \widehat{\mathbf{R}} \\
\mathbf{m}_{m}^{\prime}=a_{m}\left(\mathbf{r}_{m}^{c-}-\mathbf{r}_{m}^{c+}\right) .
\end{aligned}
$$

In (10)-(12), $\mathbf{R}=\mathbf{r}_{m}-\mathbf{r}_{n}, R=|\mathbf{R}|$, and $\widehat{\mathbf{R}}=\mathbf{R} / R$. In addition, $\mathbf{r}_{n}=\left(\mathbf{r}_{n}^{c-}+\mathbf{r}_{n}^{c+}\right) / 2$ and $\mathbf{r}_{m}=\left(\mathbf{r}_{m}^{c-}+\mathbf{r}_{m}^{c+}\right) / 2 . \mathbf{r}_{n}^{c \pm}\left(\mathbf{r}_{m}^{c \pm}\right)$ is the position vector of the centroid of $T_{n}^{ \pm}\left(T_{m}^{ \pm}\right)$. According to [8-10], the EDM can be applied when the distance between the source and the field dipole is greater than $0.15 \lambda_{g}\left(\lambda_{g}\right.$ is the wavelength in dielectric). It should be noted that $\lambda_{g}$ is the maximum wavelength in dielectric when the medium is inhomogeneous.

In $[12,13]$, the FDM is developed to efficiently calculate the interactions between far groups. In order to describe how the FDM works for dielectric and magnetic materials, we 


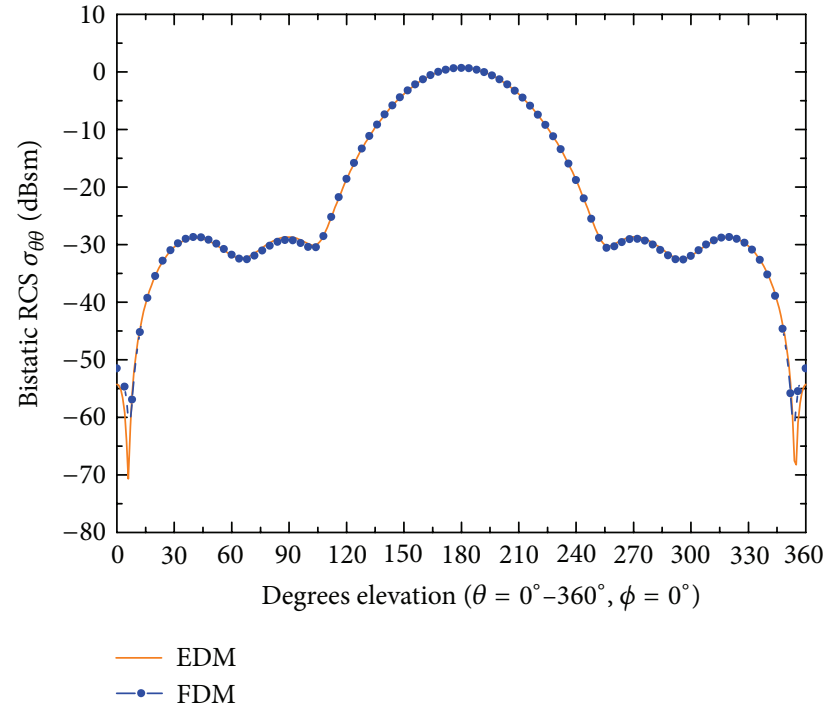

Figure 1: Bistatic RCSs ( $\theta \theta$ polarization) of a thin plate with $1.0 \mathrm{~m} \times$ $1.0 \mathrm{~m} \times 0.05 \mathrm{~m}$ and $\varepsilon_{r}=2, \mu_{r}=2$ illuminated by a plane wave with the incident direction of $(\theta, \phi)=\left(0^{\circ}, 0^{\circ}\right)$ at $0.3 \mathrm{GHz}$.

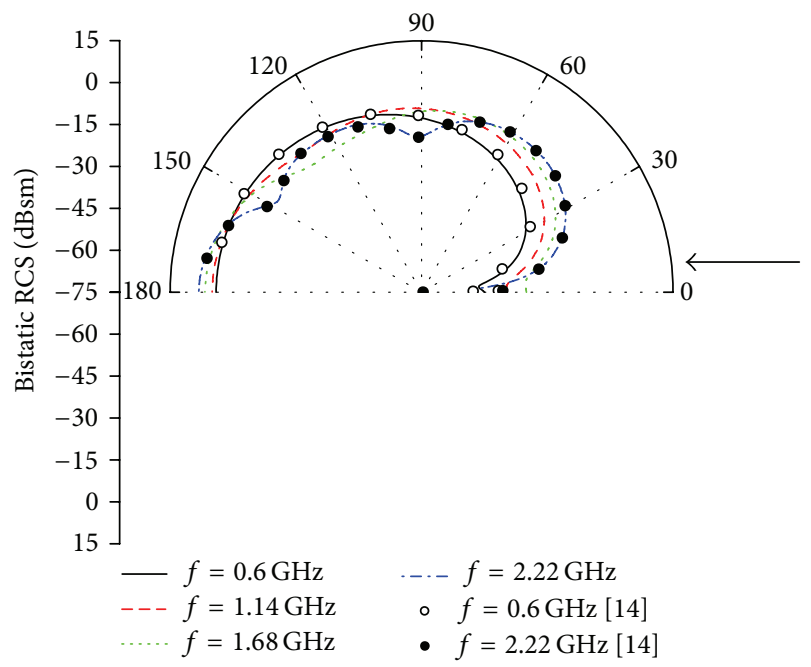

FIGURE 2: Bistatic RCS (E-plane) of an LHM sphere with radius $10 \mathrm{~cm}$ and $\varepsilon_{r}=\mu_{r}=-1-j 0.001$ operating at frequencies of $0.6 \mathrm{GHz}$, $1.14 \mathrm{GHz}, 1.68 \mathrm{GHz}$, and $2.22 \mathrm{GHz}$. The arrow indicates the incidence direction of a plane wave.

consider two dipoles $m$ and $n$, which belong to groups $j$ and $i$, respectively and suppose the two groups are a far group pair. The distance between the two dipoles can be written as $\mathbf{R}=\mathbf{r}_{m}-\mathbf{r}_{n}=\mathbf{r}_{j i}+\mathbf{r}_{m j}+\mathbf{r}_{n i}$, where $\mathbf{r}_{j i}=\mathbf{r}_{o_{j}}-\mathbf{r}_{o_{i}}, \mathbf{r}_{m j}=\mathbf{r}_{m}-\mathbf{r}_{o_{j}}$, and $\mathbf{r}_{n i}=\mathbf{r}_{n}-\mathbf{r}_{o_{i}}, \mathbf{r}_{o_{j}}$, and $\mathbf{r}_{o_{i}}$ is the center vectors of the

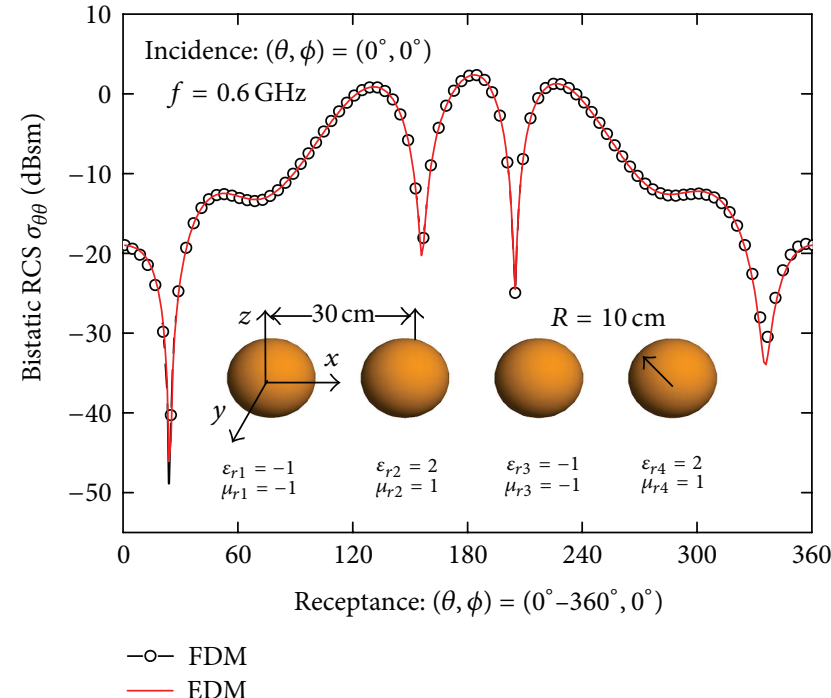

FIGURE 3: Bistatic RCS of a sphere array with $\varepsilon_{r 1}=-1, \mu_{r 1}=-1, \varepsilon_{r 2}=$ $2, \mu_{r 2}=1, \varepsilon_{r 3}=-1, \mu_{r 3}=-1, \varepsilon_{r 4}=2$, and $\mu_{r 4}=1$ and performed at $0.6 \mathrm{GHz}$ excited by a plane wave with the incident direction of $(\theta, \phi)=\left(0^{\circ}, 0^{\circ}\right)$.

groups. According to [13], $R^{\alpha}$ in the formulae (10) and (11) can be approximated using Taylor series expansion:

$$
\begin{aligned}
R^{\alpha}=\left|\mathbf{r}_{j i}+\mathbf{r}_{m j}+\mathbf{r}_{n i}\right|^{\alpha} & \\
=r_{j i}^{\alpha} & {\left[1+\left(\frac{2 \widehat{\mathbf{r}}_{j i} \cdot \mathbf{r}_{m j}}{r_{j i}}+\frac{r_{m j}^{2}}{r_{j i}^{2}}\right)+\left(\frac{2 \widehat{\mathbf{r}}_{i j} \cdot \mathbf{r}_{n i}}{r_{i j}}+\frac{r_{n i}^{2}}{r_{i j}^{2}}\right)\right.} \\
& \left.\quad-\frac{2 \mathbf{r}_{m j} \cdot \mathbf{r}_{n i}}{r_{j i}^{2}}\right]^{\alpha / 2} . \\
\approx & R_{m}^{(\alpha)}+R_{n}^{(\alpha)},
\end{aligned}
$$

where

$$
\begin{aligned}
& R_{m}^{(\alpha)}=r_{j i}^{\alpha}\left[\frac{1}{2}+\alpha\left(\frac{\widehat{\mathbf{r}}_{j i} \cdot \mathbf{r}_{m j}}{r_{j i}}+\frac{r_{m j}^{2}+(\alpha-2)\left(\widehat{\mathbf{r}}_{j i} \cdot \mathbf{r}_{m j}\right)^{2}}{2 r_{j i}^{2}}\right)\right] \\
& R_{n}^{(\alpha)}=r_{i j}^{\alpha}\left[\frac{1}{2}+\alpha\left(\frac{\widehat{\mathbf{r}}_{i j} \cdot \mathbf{r}_{n i}}{r_{i j}}+\frac{r_{n i}^{2}+(\alpha-2)\left(\widehat{\mathbf{r}}_{i j} \cdot \mathbf{r}_{n i}\right)^{2}}{2 r_{i j}^{2}}\right)\right] .
\end{aligned}
$$

And $\widehat{\mathbf{R}} \widehat{\mathbf{R}}$ can be approximated as

$$
\widehat{\mathbf{R R}} \approx \overline{\mathbf{T}}_{m}+\overline{\mathbf{T}}_{n}
$$


where

$$
\begin{gathered}
\overline{\mathbf{T}}_{m}=\frac{1}{r_{j i}^{2}}\left[\frac{1}{2} \mathbf{r}_{j i} \mathbf{r}_{j i}+\mathbf{s}_{m} \mathbf{r}_{j i}+\mathbf{r}_{j i} \mathbf{s}_{m}\right] \\
\overline{\mathbf{T}}_{n}=\frac{1}{r_{i j}^{2}}\left[\frac{1}{2} \mathbf{r}_{i j} \mathbf{r}_{i j}+\mathbf{s}_{n} \mathbf{r}_{i j}+\mathbf{r}_{i j} \mathbf{s}_{n}\right] \\
\mathbf{s}_{m}=\mathbf{r}_{m j}-\mathbf{r}_{m j} \cdot \widehat{\mathbf{r}}_{j i} \widehat{\mathbf{r}}_{j i} \\
\mathbf{s}_{n}=\mathbf{r}_{n i}-\mathbf{r}_{n i} \cdot \widehat{\mathbf{r}}_{i j} \widehat{\mathbf{r}}_{i j} .
\end{gathered}
$$

Then substituting (13) and (15) into (9), the formulae of FDM for electromagnetic materials can be obtained as follows:

$$
\begin{aligned}
& Z_{m n}^{u u} \approx \frac{j k \eta}{4 \pi} \mathbf{M}_{m}^{\prime} \cdot\left[\overline{\mathbf{I}}\left(A_{m}+A_{n}\right)\right. \\
& \left.-\left(\overline{\mathbf{T}}_{m}+\overline{\mathbf{T}}_{n}\right)\left(B_{m}+B_{n}\right)\right] \cdot \mathbf{M}_{n}^{u} \\
& =\frac{j k \eta}{4 \pi}\left[\left(A_{m} \mathbf{M}_{m}^{\prime}-B_{m} \mathbf{M}_{m}^{\prime} \cdot \overline{\mathbf{T}}_{m}\right) \cdot \mathbf{M}_{n}^{u}\right. \\
& +\mathbf{M}_{m}^{\prime} \cdot\left(A_{n} \mathbf{M}_{n}^{u}-B_{n} \overline{\mathbf{T}}_{n} \cdot \mathbf{M}_{n}^{u}\right) \\
& -\left(\mathbf{M}_{m}^{\prime} \cdot \overline{\mathbf{T}}_{m}\right) \cdot\left(B_{n} \mathbf{M}_{n}^{u}\right) \\
& \left.-\left(B_{m} \mathbf{M}_{m}^{\prime}\right) \cdot\left(\overline{\mathbf{T}}_{n} \cdot \mathbf{M}_{n}^{u}\right)\right] \text {, } \\
& u=e, m, \\
& Z_{m n}^{m e} \approx \frac{j k \eta}{4 \pi} \mathbf{M}_{m}^{\prime} \cdot\left(C_{m}+C_{n}\right)\left(\mathbf{r}_{m p}-\mathbf{r}_{n p}\right) \times \mathbf{M}_{n}^{e} \\
& =\frac{j k \eta}{4 \pi}\left[\left(C_{m} \mathbf{M}_{m}^{\prime} \times \mathbf{r}_{m p}\right) \cdot \mathbf{M}_{n}^{e}\right. \\
& +\left(\mathbf{M}_{m}^{\prime} \times \mathbf{r}_{m p}\right) \cdot\left(C_{n} \mathbf{M}_{n}^{e}\right), \\
& +\left(C_{m} \mathbf{M}_{m}^{\prime}\right) \cdot\left(\mathbf{M}_{n}^{e} \times \mathbf{r}_{n p}\right) \\
& \left.+\mathbf{M}_{m}^{\prime} \cdot\left(C_{n} \mathbf{M}_{n}^{e} \times \mathbf{r}_{n p}\right)\right] \text {, } \\
& Z_{m n}^{e m} \approx-\frac{j k \eta}{4 \pi} \mathbf{M}_{m}^{\prime} \cdot\left(C_{m}+C_{n}\right)\left(\mathbf{r}_{m p}-\mathbf{r}_{n p}\right) \times \mathbf{M}_{n}^{m} \\
& =-\frac{j k \eta}{4 \pi}\left[\left(C_{m} \mathbf{M}_{m}^{\prime} \times \mathbf{r}_{m p}\right) \cdot \mathbf{M}_{n}^{m}\right. \\
& +\left(\mathbf{M}_{m}^{\prime} \times \mathbf{r}_{m p}\right) \cdot\left(C_{n} \mathbf{M}_{n}^{m}\right), \\
& +\left(C_{m} \mathbf{M}_{m}^{\prime}\right) \cdot\left(\mathbf{M}_{n}^{m} \times \mathbf{r}_{n p}\right) \\
& \left.+\mathbf{M}_{m}^{\prime} \cdot\left(C_{n} \mathbf{M}_{n}^{m} \times \mathbf{r}_{n p}\right)\right],
\end{aligned}
$$

where $\mathbf{r}_{m p}=\mathbf{r}_{m}-\mathbf{r}_{p}, \mathbf{r}_{n p}=\mathbf{r}_{n}-\mathbf{r}_{p}, \mathbf{r}_{p}=\left(\mathbf{r}_{o_{j}}+\mathbf{r}_{o_{i}}\right) / 2$,

$$
\begin{gathered}
A_{u}=R_{u}^{(-1)}+(j k)^{-1} R_{u}^{(-2)}+(j k)^{-2} R_{u}^{(-3)}, \\
B_{u}=R_{u}^{(-1)}+3(j k)^{-1} R_{u}^{(-2)}+3(j k)^{-2} R_{u}^{(-3)}, \\
C_{u}=R_{u}^{(-2)}+(j k)^{-1} R_{u}^{(-3)}, \\
\mathbf{M}_{m}^{\prime}=\mathbf{m}_{m}^{\prime} e^{-j k R_{m}^{(1)}}, \\
\mathbf{M}_{n}^{u}=\mathbf{m}_{n}^{u} e^{-j k R_{n}^{(1)}}, \quad u=e, m .
\end{gathered}
$$

In the following part, we will present how to use the FDM to calculate the interactions among the equivalent electric or magnetic dipoles in group $i$ and group $j$. The effect of all the electric and magnetic dipoles in group $i$ on the $m$ th electric dipoles in group $j$ can be expressed as

$$
\begin{aligned}
\sum_{n \in i}\left(Z_{m n}^{e e} I_{n}^{e}+Z_{m n}^{e m} I_{n}^{m}\right) & \\
\approx \frac{j k \eta}{4 \pi}[ & \left(A_{m} \mathbf{M}_{m}^{\prime}-B_{m} \mathbf{M}_{m}^{\prime} \cdot \overline{\mathbf{T}}_{m}\right) \cdot \sum_{n \in i} I_{n}^{e} \mathbf{M}_{n}^{e} \\
& +\mathbf{M}_{m}^{\prime} \cdot \sum_{n \in i} I_{n}^{e}\left(A_{n} \mathbf{M}_{n}^{e}-B_{n} \overline{\mathbf{T}}_{n} \cdot \mathbf{M}_{n}^{e}\right) \\
& -\left(\mathbf{M}_{m}^{\prime} \cdot \overline{\mathbf{T}}_{m}\right) \cdot \sum_{n \in i} I_{n}^{e} B_{n} \mathbf{M}_{n}^{e} \\
& -\left(B_{m} \mathbf{M}_{m}^{\prime}\right) \cdot \sum_{n \in i} I_{n}^{e} \overline{\mathbf{T}}_{n} \cdot \mathbf{M}_{n}^{e} \\
& -\left(C_{m} \mathbf{M}_{m}^{\prime} \times \mathbf{r}_{m p}\right) \cdot \sum_{n \in i} I_{n}^{m} \mathbf{M}_{n}^{m} \\
& -\left(\mathbf{M}_{m}^{\prime} \times \mathbf{r}_{m p}\right) \cdot \sum_{n \in i} I_{n}^{m} C_{n} \mathbf{M}_{n}^{m} \\
& -\left(C_{m} \mathbf{M}_{m}^{\prime}\right) \cdot \sum_{n \in i} I_{n}^{m} \mathbf{M}_{n}^{m} \times \mathbf{r}_{n p} \\
& \left.-\mathbf{M}_{m}^{\prime} \cdot \sum_{n \in i} I_{n}^{m} C_{n} \mathbf{M}_{n}^{m} \times \mathbf{r}_{n p}\right]
\end{aligned}
$$

In a similar way, the effect of all the electric and magnetic dipoles in group $i$ on the $m$ th magnetic dipoles in group $j$ can be expressed as

$$
\begin{aligned}
\sum_{n \in i}\left(Z_{m n}^{m m} I_{n}^{m}+Z_{m n}^{m e} I_{n}^{e}\right) & \\
\approx \frac{j k \eta}{4 \pi}[ & \left(A_{m} \mathbf{M}_{m}^{\prime}-B_{m} \mathbf{M}_{m}^{\prime} \cdot \overline{\mathbf{T}}_{m}\right) \cdot \sum_{n \in i} I_{n}^{m} \mathbf{M}_{n}^{m} \\
& +\mathbf{M}_{m}^{\prime} \cdot \sum_{n \in i} I_{n}^{m}\left(A_{n} \mathbf{M}_{n}^{m}-B_{n} \overline{\mathbf{T}}_{n} \cdot \mathbf{M}_{n}^{m}\right) \\
& -\left(\mathbf{M}_{m}^{\prime} \cdot \overline{\mathbf{T}}_{m}\right) \cdot \sum_{n \in i} I_{n}^{m} B_{n} \mathbf{M}_{n}^{m} \\
& -\left(B_{m} \mathbf{M}_{m}^{\prime}\right) \cdot \sum_{n \in i} I_{n}^{m} \overline{\mathbf{T}}_{n} \cdot \mathbf{M}_{n}^{m}
\end{aligned}
$$




$$
\begin{aligned}
& +\left(C_{m} \mathbf{M}_{m}^{\prime} \times \mathbf{r}_{m p}\right) \cdot \sum_{n \in i} I_{n}^{e} \mathbf{M}_{n}^{e} \\
& +\left(\mathbf{M}_{m}^{\prime} \times \mathbf{r}_{m p}\right) \cdot \sum_{n \in i} I_{n}^{e} C_{n} \mathbf{M}_{n}^{e} \\
& +\left(C_{m} \mathbf{M}_{m}^{\prime}\right) \cdot \sum_{n \in i} I_{n}^{e} \mathbf{M}_{n}^{e} \times \mathbf{r}_{n p} \\
& \left.+\mathbf{M}_{m}^{\prime} \cdot \sum_{n \in i} I_{n}^{e} C_{n} \mathbf{M}_{n}^{e} \times \mathbf{r}_{n p}\right] .
\end{aligned}
$$

It can be found that each term in (19) and (20) achieves the separation of $m$ and $n$. Therefore, the summation results $\sum_{n \in i}(\cdot)$ in (19) and (20) are independent of $m$ and can be reused by each dipole in group $j$. These summation results $\sum_{n \in i}(\cdot)$ only need to be computed once. If we suppose that groups $i$ and $j$ contain $N_{i}$ and $N_{j}$ electric or magnetic dipoles, respectively, then the complexity of interactions between the two far groups is reduced from $O\left(N_{i} N_{j}\right)$ to $O\left(N_{i}+N_{j}\right)$.

\section{Numerical Results and Discussion}

All the simulations are performed on a personal computer with the Intel Pentium Dual-Core CPU E2200 with $2.0 \mathrm{GHz}$ (only one core was used) and 2.0 GB RAM. The GMRES iterative solver is employed to obtain an identical residual error, 0.001 .

First, we consider the scattering problem of a thin plate with $1.0 \mathrm{~m} \times 1.0 \mathrm{~m} \times 0.05 \mathrm{~m}$ and $\varepsilon_{r}=2, \mu_{r}=2$. The plate is divided into 2665 volumetric cells with an average edge length of $0.05 \mathrm{~m}$, and the total number of unknowns is 12420 . Totally, 256 nonempty groups with the size of 0.2 are obtained. The bistatic RCS in $\theta \theta$ polarization is compared with the EDM shown in Figure 1. Both the CPU time and memory requirements are shown in Table 1 . We can see from Table 1, it costs 185 seconds and $312 \mathrm{MB}$ of memory using the FDM, whereas 674 seconds and $1239 \mathrm{MB}$ of memory are needed using the EDM. That is to say the FDM yields reductions of $72.6 \%$ of the total computation time and $74.8 \%$ of the memory requirements using EDM.

Furthermore, we consider the scattering problem of a $10 \mathrm{~cm}$ sphere with $\varepsilon_{r}=\mu_{r}=-1-j 0.001$ as an example. To validate our FDM method, the calculated frequencies span the range from $0.6 \mathrm{GHz}$ to $2.22 \mathrm{GHz}$. Figure 2 gives the bistatic RCSs for E-plane calculated by FDM. The results are in agreement with those from [14] (the RCSs at frequencies of $0.6 \mathrm{GHz}$ and $2.22 \mathrm{GHz}$ in [14] have been shown in Figure 2 as references). We compared the CPU time of the FDM with that of EDM when the object is at frequency of $1.14 \mathrm{GHz}$ in Table 1. At this frequency, the sphere is divided into 1624 volumetric cells with an average edge length of $0.03 \mathrm{~m}$, and the total number of unknowns is 6822. All the unknowns are divided into 1324 nonempty groups and the size of each group is 0.2 . We can see from Table 1 that it costs 376 seconds and $173 \mathrm{MB}$ of memory using the FDM, whereas 1753 seconds and $382 \mathrm{MB}$ of memory are needed using the EDM.
TABLE 1: Comparison of CPU time, memory cost, and iterations of RCS solutions for EDM and FDM.

\begin{tabular}{lccc}
\hline Method & Time $(\mathrm{s})$ & Memory $(\mathrm{MB})$ & Iterations \\
\hline \multicolumn{4}{c}{ Problem 1: RHM cube } \\
\hline EDM & 674 & 1239 & 13 \\
FDM & 185 & 312 & \\
\hline \multicolumn{4}{c}{ Problem 2: LHM sphere at 1.14 GHz } \\
\hline EDM & 1753 & 382 \\
FDM & 376 & 173 & 305 \\
\hline \multicolumn{5}{c}{ Problem 3: LHM-RHM sphere array } \\
FDM & 4204 & 863 \\
\hline
\end{tabular}

Finally, we consider an LHM-RHM composite array. The dimension and parameters of the array are shown in Figure 3. The geometry is discretized into 2413 tetrahedrons using $0.04 \mathrm{~m}$ average edge length, thus resulting in total 10340 unknowns. All the unknowns are divided into 1342 nonempty groups and the size of each group is 0.2 . $\theta \theta$ polarized RCS is plotted, as shown in Figure 3. It can be seen from Table 1 that it costs 435 seconds and $272 \mathrm{MB}$ of memory using the FDM, whereas 4204 seconds and $863 \mathrm{MB}$ of memory using the EDM are needed. That is to say the FDM can save much more time and memory requirements than the EDM. From Table 1, we also can find that the method converges much more slowly when it is used to analyze the scattering involving the LHM than the RHM. This is because LHM structures usually present numerical resonances that inhibit quick convergence of iterations [15].

\section{Conclusion}

In this work, the formulations of FDM for analyzing the electromagnetic scattering from electromagnetic materials are given, which saves CPU time and memory requirements compared with the conventional EDM. Several dielectric and magnetic targets include RHM objects and LHM objects, and LHM-RHM composite objects are calculated to demonstrate the merit of this method. Furthermore, the numerical results show that the convergences may become much slower if the LHM is included in the targets. In further work, we will try to construct an efficient preconditioner to alleviate the disadvantage brought by the LHM.

\section{Acknowledgments}

This work was supported by the National Nature Science Foundation of China under Grant no. 61071019, the Joint Funding Projects of the Aerospace Science Foundation Office of China no. 2008ZA52006, and a project funded by the Priority Academic Program Development of Jiangsu Higher Education Institutions. 


\section{References}

[1] M. K. Li and W. C. Chew, "Applying divergence-free condition in solving the volume integral equation," Progress in Electromagnetics Research, vol. 57, pp. 311-333, 2006.

[2] X. M. Pan, W. C. Pi, and X. Q. Sheng, "On openmp parallelization of the multilevel fast multipole algorithm," Progress in Electromagnetics Research, vol. 112, pp. 199-213, 2011.

[3] W. Wang and N. Nishimura, "Calculation of shape derivatives with periodic fast multipole method with application to shape optimization of metamaterials," Progress in Electromagnetics Research, vol. 127, pp. 49-64, 2012.

[4] X. M. Pan, L. Cai, and X. Q. Sheng, "An efficient high order multilevel fast multipole algorithm for electromagnetic scattering analysis," Progress in Electromagnetics Research, vol. 126, pp. 85100, 2012.

[5] E. Bleszynski, M. Bleszynski, and T. Jaroszewicz, "AIM: adaptive integral method for solving large-scale electromagnetic scattering and radiation problems," Radio Science, vol. 31, no. 5, pp. 1225-1251, 1996.

[6] L. Hu, L. W. Li, and T. S. Yeo, "Analysis of scattering by large inhomogeneous bi-anisotropic objects using AIM," Progress in Electromagnetics Research, vol. 99, pp. 21-36, 2009.

[7] X. Q. Zhu, Y. L. Geng, and X. B. Wu, "Application of MoMCGM-FFT method to scattering from three-dimensional magnetic anisotropic scatterers," Chinese Journal of Radio Science, vol. 17, no. 3, pp. 209-215, 2002.

[8] J. Yuan, C. Gu, and G. Han, "Efficient generation of method of moments matrices using equivalent dipole-moment method," IEEE Antennas and Wireless Propagation Letters, vol. 8, pp. 716719, 2009.

[9] J. Yuan, Z. Niu, Z. Li, and C. Gu, "Electromagnetic scattering by arbitrarily shaped PEC targets coated with anisotropic media using equivalent dipole-moment method," Journal of Infrared, Millimeter, and Terahertz Waves, vol. 31, no. 6, pp. 744-752, 2010.

[10] X. Deng, C. Gu, and Y. Zhou, "Electromagnetic scattering by arbitrary shaped three-dimensional conducting objects covered with electromagnetic anisotropic materials," ACES Journal, vol. 26, no. 11, pp. 886-892, 2011.

[11] D. H. Schaubert, D. R. Wilton, and A. W. Glisson, "A tetrahedral modeling method for electromagnetic scattering by arbitrarily shaped inhomogeneous dielectric bodies," IEEE Transactions on Antennas and Propagation, vol. 32, no. 1, pp. 77-85, 1984.

[12] X. Chen, Z. Niu, Z. Li, and C. Gu, "A hybrid fast dipole method and adaptive modified characteristic basis function method for electromagnetic scattering from perfect electric conduction targets," Journal of Electromagnetic Waves and Applications, vol. 25, no. 14-15, pp. 1940-1952, 2011.

[13] X. Chen, Z. Li, Z. Niu, and C. Gu, "Analysis of electromagnetic scattering from PEC targets using improved fast dipole method," Journal of Electromagnetic Waves and Applications, vol. 25, no. 16, pp. 2254-2263, 2011.

[14] C. Monzon, "Scattering properties of an impedance-matched, ideal, homogeneous, causal "left-handed" sphere," The Journal of the Optical Society of America, vol. 21, no. 12, pp. 2311-2319, 2004.

[15] L. Gürel, Ö. Ergül, A. Ünal, and T. Malas, "Fast and accurate analysis of metamaterial structures using the multilevel fast multipole algorithm," Progress in Electromagnetics Research, vol. 95, pp. 179-198, 2009. 

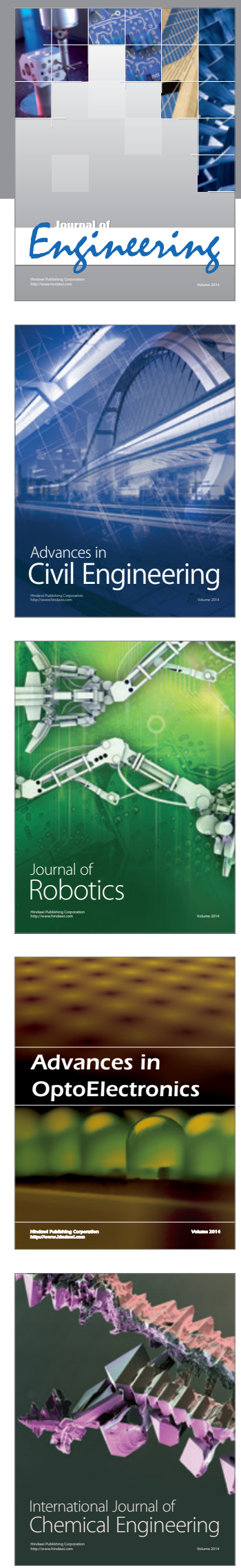

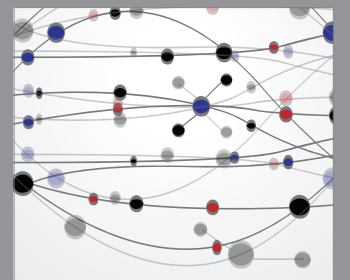

The Scientific World Journal
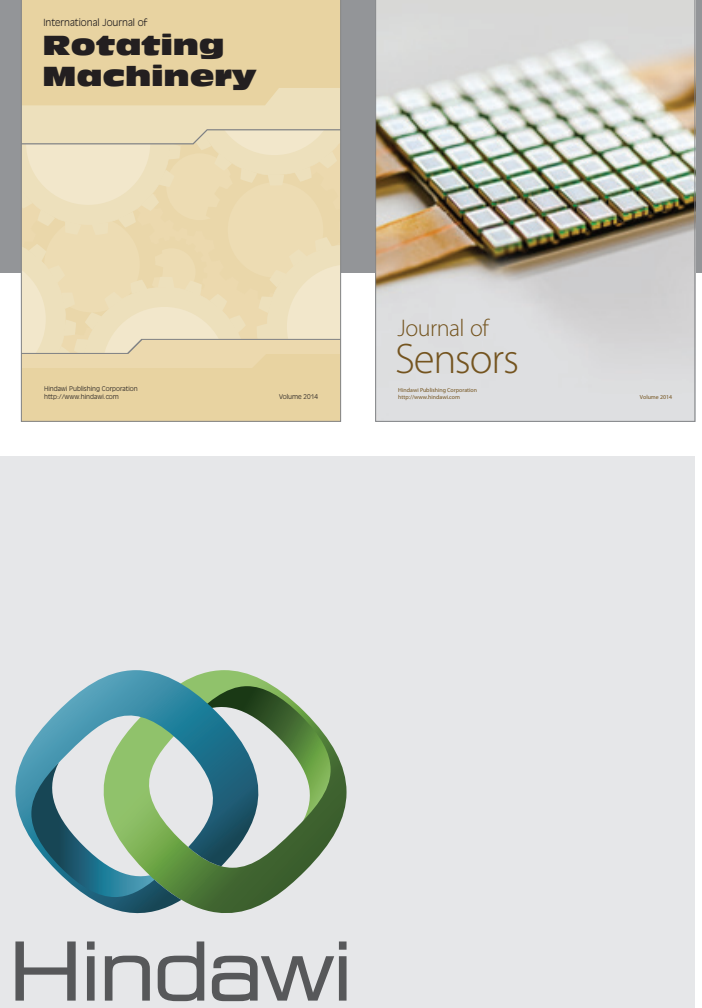

Submit your manuscripts at http://www.hindawi.com
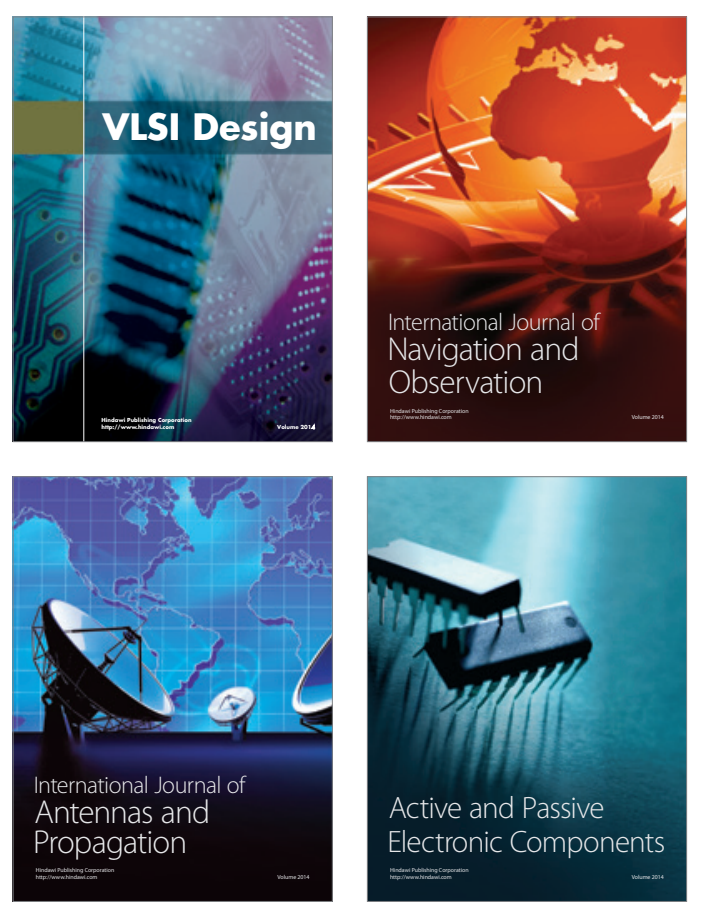
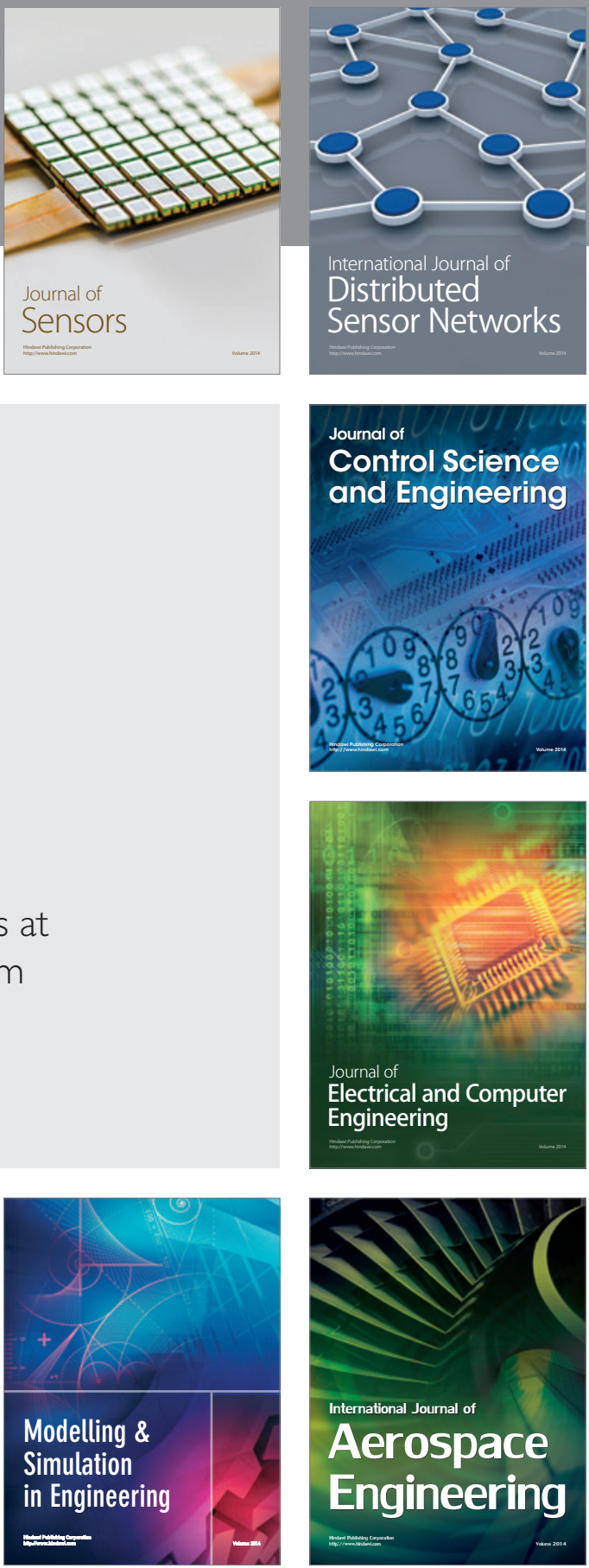

Journal of

Control Science

and Engineering
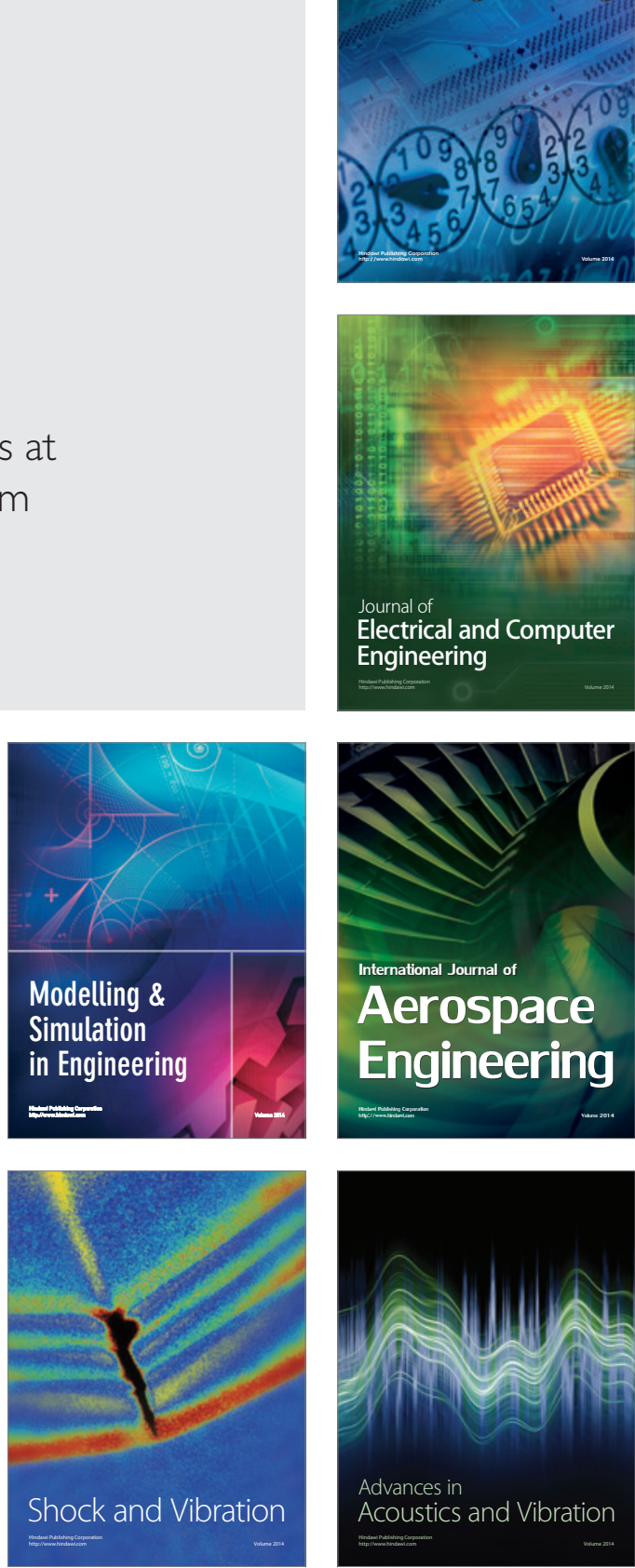